\title{
Design Concept of Tissue-Engineered Products for Use in Emergency and Critical Care Medicine
}

\author{
Misato KUROYANAGI ${ }^{1}$ and Yoshimitsu KUROYANAGI ${ }^{2,3 *}$ \\ ${ }^{1}$ Department of Emergency and Critical Care Medicine, Nippon Medical School Chiba Hokusoh Hospital, Inzai, Chiba, Japan \\ ${ }^{2}$ Kitasato University, Minami-ku, Sagamihara, Kanagawa, Japan \\ ${ }^{3}$ Technosurg Ltd., Midori-ku, Sagamihara, Kanagawa, Japan
}

Received: January 20, 2018; Published: January 25, 2018

*Corresponding author: Yoshimitsu Kuroyanagi, Emeritus Professor of Kitasato University, Technosurg Ltd., 5-4-30, Nishihashimoto, Midori-ku, Sagamihara, Kanagawa, Japan Email: drs.yoshi@technosurg.co.jp

\begin{abstract}
Three types of tissue-engineered products were developed using cell, growth factor, and biomaterial. The first product is a CDS composed of allogeneic fibroblasts combined with a HA and Col spongy sheet. Fibroblast can release various growth factors that are essential for wound healing. HA and Col have a potential to enhance wound healing. This CDS can be cryopreserved for a long period of up to clinical use. The clinical study demonstrated that allogeneic CDS is a useful product. The second product is a wound dressing composed of HA and Col containing EGF. EGF has a potential to enhance wound healing. This wound dressing is a freeze-dried spongy sheet, thereby the efficacy of EGF can be kept for a long period of up to clinical use. The clinical study showed that EGF-incorporating wound dressing is a practical product. The third product is an anti-adhesive spongy sheet composed of HA and Col containing EGF. This is also a freeze-dried spongy sheet. The animal study indicated that EGF-incorporating anti-adhesive spongy sheet is a promissing product.
\end{abstract}

Abbreviations: CES: Cultured Epidermal Substitute, CDS: Cultured Dermal Substitute, CSS: Cultured Skin Substitute, HA: Hyarulonic Acid, Col: Collagen, EGF: Epidermal Growth Factor, VEGF: Vascular Endothelial Growth Factor, HGF: Hepatocyte Growth Factor, bFGF: basic Fibroblast Growth Factor

\section{Introduction}

The tissue-engineered products include three prime constituents, namely cell, growth factor, and biomaterial. Understanding the wound healing processe provides useful information for the design concept of tissue-engineered products [1-3]. Various types of tissueengieered products using cells with or without biomaterial have been developed for the treatment of burn injury. CES is composed of stratified keratinocytes. CDS is composed of fibroblasts combined with a scaffold of biomaterial. CSS is composed of fibroblasts combined with a scaffold of biomaterial bearing stratified keratinocytes. They are classified into two categories depending on the use of autologous cells or allogeneic cells. Autologous CES has been proved very useful [4-6]. Allogeneic CSS also has been proved very useful [7]. We developed CDS composed of fibroblasts combined with a HA and Col spongy sheet [8]. Fibroblast can release various growth factors such as VEGF, HGF, and bFGF that are essential for wound healing $[9,10]$. $\mathrm{HA}$ and Col have a potential to enhance wound healing. HA is a useful biomaterial for wound healing, as it has various biological activities [11,12]. HA is able to create an excellent wound healing environment. HA also stimulates cell migration and angiogenesis. Col is also a useful biomaterial for wound healing. Col-derived peptides are able to act as a chmo-attractant for fibroblasts in vitro and may have a similar activity in vivo [13]. The clinical study demonstrated that allogeneic CDS is a useful product [14].

Based on the design concept of CDS, we developed EGFincorporating wound dressing $[15,16]$. EGF has a potential to enhance proliferation of keratinocyte and fibroblast, and also to stimulate fibroblast to synthesize an increased amount of VEGF and HGF that are essential for angiogenesis $[17,18]$. This freeze-dried wound dressing is very practical for the treatment of burn injury. The clinical study showed that EGF-incorporating wound dressing is a practical product [19]. Based on the design concept of EGFincorporating wound dressing, we developed EGF-incorporating anti-adhesive product [20]. During surgical opperation, incision is necessary for the excision of target tissues or organs. Incision wounds result in a tissue-adhesion among incision wound, 
surgically excised wound, and surrounding organs. To prevent undesirable tissue-adhesion, some biodegradable anti-adhesive products are applied among these wounds and surrounding organs. The animal study using rats indicated that EGF-incorporating antiadhesive spongy sheet is a promissing product.

Wound dressing is applied to external wounds, while anti- adhesive product is applied to internal wounds. Both products should be designed to have a common function that facilitates wound healing. In more successful strategy for anti-adhesive products, two main events must proceed simultaneously: facilitating wound healing of surgically excised wound and preventing adhesion among these wounds and surrounding organs (Figure 1).

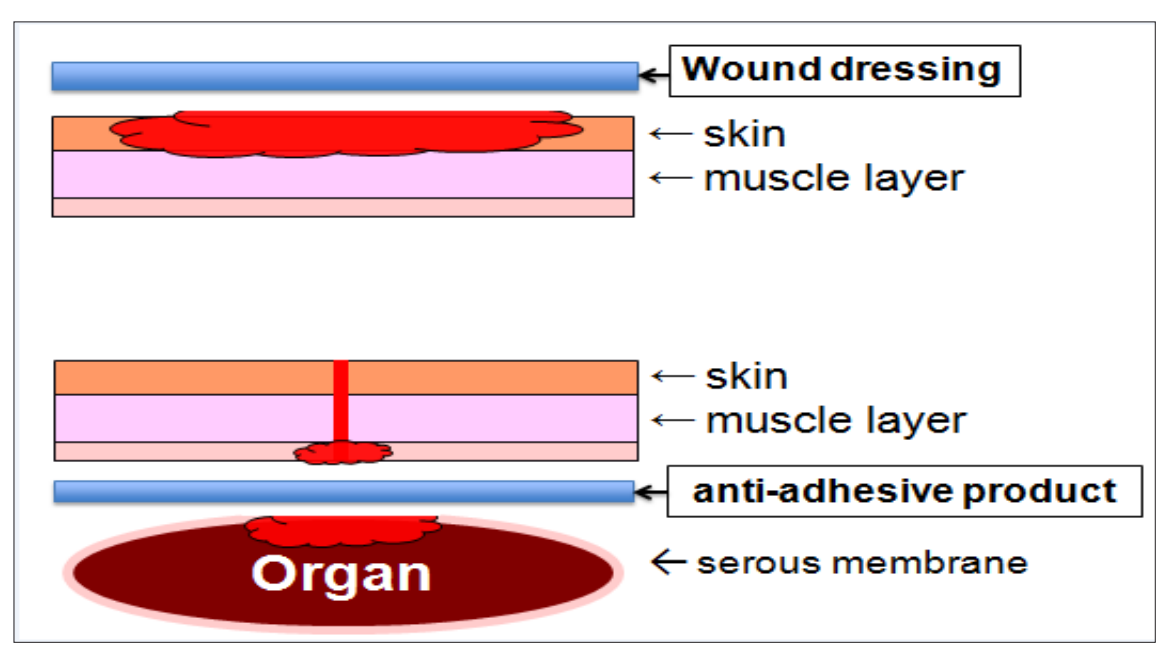

Figure 1: Design concept of wound dressing and anti-adhesive product: wound dressing is applied to external wounds, while anti-adhesive product is applied to internal wounds. Both products should be designed to have a common function that facilitates wound healing. In more successful strategy for anti-adhesive products, two main events must proceed simultaneously: facilitating wound healing of surgically excised wound and also preventing adhesion among these wounds and surrounding organs.

\section{Allogeneic Cultured Dermal Substitue}

Allogeneic CDS is manufactured by seeding fibroblasts on the Col layer of two-layered spogny sheet of HA and Col, followed by culturing for 1 week. This spongy sheet has an intermolecular crosslinking among HA and Col molecules, and therefore this CDS is able to be cryopreserved for a long period and then thawed prior to clinical application. The cryopreserved CDS is able to keep the potential to release VEGF, HGF, and bFGF at appreciable levels. In practice, allogeneic CDS is manufactured and cryopreserved at $-80^{\circ} \mathrm{C}$ below. The cryopreserved CDS is delivered to other hospitals using a box filled with dry ice and then continuingly cryopresereved at $-80^{\circ} \mathrm{C}$ below. The efficacy of CDS was assessed in 415 cases, including severe burns and intractable skin ulcers. Excellent and good results were assessed in $92.6 \%$ of the all cases in total clinical evaluation [3].

\section{EGF-Incorporating-Wound Dressing}

EGF has some useful effects for wound healing. HA and Col have also some useful effects for wound healing. EGF-incorprating wound dressing was manufactured by freeze-drying an aqueous solution of high molecular weight HA, low molecular weight HA, and Col containing EGF. Both side of spongy sheet was irradiated with an ultraviolet lamp in order to induce intermolecular cross linking among Col molecules. This wound dressing is designed to contain EGF at a concentration of $2.0 \mu \mathrm{g} / \mathrm{cm}^{2}$. The efficacy of EGFincorporating wound dressing was assessed in 16 clinical cases, incuding burns, donor sites, traumatic skin defects, and intractable skin ulcers. This treatment using EGF-wound dressing was applied repeatedly at an interval of 3 to 5 days for a period of 6 weeks, or longer if necessary. Excellent and good results were assessed in $93.7 \%$ of the all cases in total clinical evaluation [19].

\section{EGF-Incorporating Anti-Adhesive Product}

EGF-incorprating anti-adhesinve product was prepared by freeze-drying an aqueous solution of high molecular weight HA, low molecular weight HA, Col, and EGF. This anti-adhesinve product is designed to contain EGF at a concentration of $1.0 \mu \mathrm{g} /$ $\mathrm{cm}^{2}$. The potential of this anti-adhesive product was evaluated using an inter-tissue model. Briefly, in order to create an intertissue model, a fibroblast-incorporating collagen gel sheet was placed on a stainless mesh in a dish containing conventional culture medium. A piece of anti-adhesive spongy sheet was placed on the fibroblast-incorporating collagen gel sheet, on which another fibroblast-incorporating collagen gel sheet was placed. Production of VEGF and HGF by fibroblasts was measured. This experiment demonstrated that EGF-incorporating anti-adhesive product effectively stimulates fibroblasts to release an increased amount of VEGF and HGF. The anti-adhesive performance was evaluated in animal study using rats. Briefly, a midline incision through the abdominal wall was made under intraperitonal pentobarbital sodium anesthesia. A surface of cecum was abraded by scraping with a scalpel blade. A surface of peritoneum was superficially excised. Both cecum defect and peritoneal defect were exposed to air for $10 \mathrm{~min}$ in order to induce additional tissue damage by 
dehydration. A piece of EGF-incorporating anti-adhesive spongy sheet was placed on the peritoneal defect. In control, no product was placed on the peritoneal defect. The findings in this animal test indicated that EGF-incorporating anti-adhesive spongy sheet is capable of facilitating wound healing of surgically excised wound and preventing surgically excised wound from adhering to surrounding organs [20].

\section{Conclusion}

Autologous CES needs manufacturing time more than 3 weeks, therefore it is not practical for use in emergency and critical medicine. Allogeneic CSS manufactured by using a collagen gel sheet is not able to be cryopreserved, because of its handling unstability in the freezing and thawing process. Therefore, it is not practical for use in emergency and critical medicine. On the contrary, allogeneic CDS manufactured by using a HA and Col spongy sheet with intermorecular cross linking is able to be cryopreserved, because of its sufficient handling stability in the freezing and thawing process. This CDS is able to be cryopreserved for a long period up to clinical use and then thawed prior to clinical application. Therefore, it is practical for use in emergency and critical medicine. In addition, freeze-dried products such as EGF-incorprating wound dressing and EGF-incorporating anti-adhesinve product are more practical for use in emergency and critical medicine, because of simple operation of only removed from the sterile bag. In conclusion, the efficacy of tissue-engineered product using allogeneic cell, growth factor, and biomaterial is dependent on the potential of growth factor and biomaterial to facilitate wound healing.

\section{References}

1. Kuroyanagi Y (2016) Tissue-engineered product for skin regenerative medicine. Open J Regenerative Med 5(3): 61-84.

2. Kuroyanagi M, Kuroyanagi Y (2017) Tissue-enginerred products capable of enhancing wound healing. AIMS Materials Science 4(3): 561-581.

3. Kuroyanagi M, Kuroyanagi Y (2018) Advanced treatment of burns and skin ulcers using tissue-engineered products. Dermatology Case Reports 3: $137-142$.

4. O'Connor NE, Muliken JG, Banks Schlegel S, Keinde O, Green H (1982) Grafting of burns with cultured epithelium prepared from autologous epidermal cells. Lancet 1: 75-78.

5. Gallico GG, O'Connor NE, Compton CC, Kehinde O, Green H (1984) Permanent coverage of large burn wounds with autologous cultured human epithelium. N Engl J Med 311(7): 448-451.
6. Odessey R (1992) Multicenter experience with cultured epidermal autograft for treatment of burns. J Burn Care Rehabil 13(1):174-180.

7. Parenteau N, Naughton G (1999) Skin; the first tissue-engineered products. Sci Am 280(4): 83-84.

8. Kuroyanagi Y, Kubo K, Matsui H, Kim HJ, Numari S, et al. (2004) Establishment of banking system for allogeneic cultured dermal substitute. Artif Organs 28(1): 13-21.

9. Kubo K, Kuroyanagi Y (2005) A study of cytokines released from fibroblasts in cultured dermal subsitute. Artifi Organs 29(10): 845-849.

10. Hashimoto A, Kuroyanagi Y (2008) Standardization for mass production of allogeneic cultured dermal substitute by measuring the amount of VEGF, bFGF, HGF, TGF- $\beta$, and IL-8. J Artif Organs 11(4): 225-231.

11. Chen WYJ, Abatangelo G (1999) Functions of hyaluronan in wound repair. Wound Repair and Regeneration 7(2): 79-89.

12. Pardue EL, Ibrahim S, Ramamurthi A (2008) Role of hyaluronan in angiogenesis and its utility to angiogenic tissue engineering. Organogenesis 4(4): 203-214.

13. Postlethwaite AE, Seyer JM, Kang AH (1978) Chemotactic attraction of human fibroblasts to type I, II, and III collagens and collagen-derived peptides. Proc Natl Acad Sci USA 75(2): 871-875.

14. Kashiwa N, Ito O, Ueda T, Kubo K, Matsui H, Kuroyanagi Y (2004) Treatment of full-thickness skin defect with concomitant grafting of 6-fold extended mesh auto-skin and allogeneic cultured dermal substitute. Artif Organs 28(5): 444-450.

15. Kondo S, Kuroyanagi Y (2012) Development of wound dressing composed of hyaluronic acid and collagen sponge with epidermal growth factor. J Biomater Sci 23(5): 629-643.

16. Kondo S, Niiyama H, Yu A, Kuroyanagi, Y (2012) Evaluation of a wound dressing composed of hyaluronic acid and collagen sponge containing epidermal growth factor in diabetic mice. J Biomateri Sci 23(13): 17291740.

17. Carpenter G, Cohen S (1976) Human epidermal growth factor and the proliferation of human fibroblasts. J Cell Physiol 88(2): 227-237.

18. Carpenter G, Cohen S (1979) Epidermal growth factor. Annu Rev Biochem 48: 193-216.

19. Yu A, Takeda A, Kumazawa K, Miyoshi H, Kuroyanagi M, et al. (2015) Preliminary clinical study using a novel wound dressing composed of hyaluronic acid and collagen containing EGF. Open J Regenerative Med 4(1): 6-13.

20. Kuroyanagi M, Yamamoto A, Shimizu N, Toi A, Inomata T, et al. (2014) Development of anti-adhesive spongy sheet composed of hyaluronic acid and collagen containing epidermal growth factor. J Biomatger Sci 25(12): 1253-1265.
This work is licensed under Creative Commons Attribution 4.0 License

Submission Link: http://biomedres.us/submit-manuscript.php

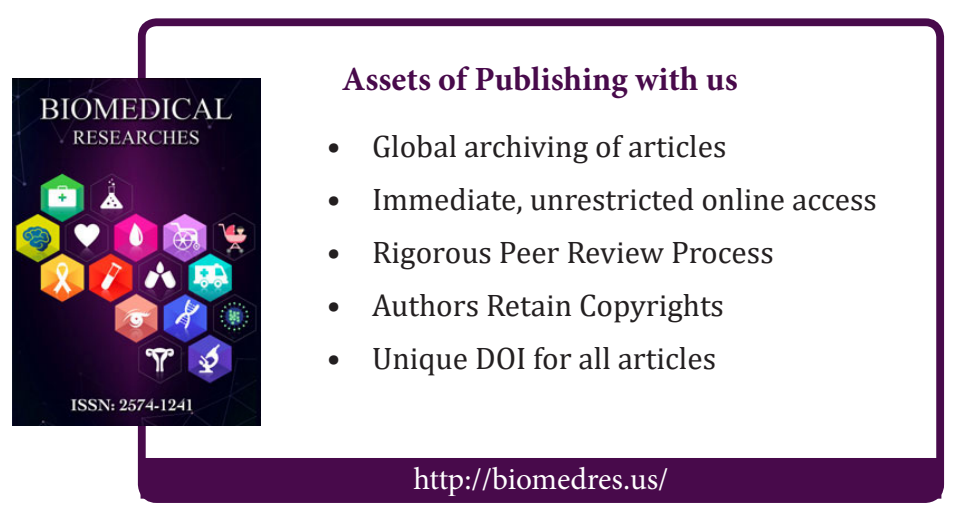

